\title{
Collaboration for Improved Disease Surveillance Literature Review
}

\author{
Tera Reynolds*1, Katie Suda ${ }^{2}$, Blaine Reeder ${ }^{3}$, William Storm ${ }^{4}$, Al Ozonoff ${ }^{5}$ and Howard \\ Burkom $^{6}$ \\ ${ }^{1}$ International Society for Disease Surveillance, Brighton, MA, USA; ${ }^{2}$ University of Tennessee, Memphis, TN, USA; ${ }^{3}$ University of \\ Washington, Seattle, WA, USA; ${ }^{4}$ Ohio Department of Health, Columbus, OH, USA; ${ }^{5}$ Boston Children's Hospital, Boston, MA, USA;

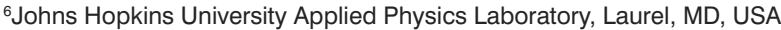

\section{Objective}

To improve the method of automated retrieval of surveillance-related literature from a wide range of indexed repositories.

\section{Introduction}

The ISDS Research Committee (RC) is an interdisciplinary group of researchers interested in various topics related to disease surveillance. The $\mathrm{RC}$ hosts a literature review process with a permanent repository of relevant journal articles and bimonthly calls that provide a forum for discussion and author engagement. The calls have led to workgroups and society-wide events, boosted interest in the ISDS Conference, and fostered networking among participants.

Since 2007, the RC has identified and classified published articles using an automated search method with the aim of progressing ISDS's mission of advancing the science and practice of disease surveillance by fostering collaboration and increasing awareness of innovations in the field of surveillance. The RC literature review efforts have provided an opportunity for interprofessional collaboration and have resulted in a repository of over 1,000 articles, but feedback from ISDS members indicated relevant articles were not captured by the existing methodology. The method of automated literature retrieval was thus refined to improve efficiency and inclusiveness of stakeholder interests.

\section{Methods}

The earlier literature review method was implemented from March 2007 to March 2012. PubCrawler [1] (articles indexed in Medline) and Google Scholar [2] search results were sent to the RC via automated e-mail. To refine this method, the RC developed search strings in PubMed [3], Embase [4], and Scopus [5], consisting of over 100 terms suggested by members. After evaluating these methods, we found that the Scopus search is the most comprehensive and improved the cross-disciplinary scope. Scopus results allowed filtering of 50-100 titles and abstracts in fewer than 30 minutes each week for the identification of relevant articles (Figure).

Journal titles were categorized to assess the increased range of fields covered; categories include epidemiology, agriculture, economics, and medicine (51 categories total)

\section{Results}

Since implementing the new method, potentially relevant articles identified per month increased from an average of 19 (SD: 13; $n=31$ ) to 159 (SD: $63 ; n=3$ ). Both methods identified articles in the health sciences, but the new search also captured articles in the life, physical, and social sciences. Between March 2007 and March 2012, articles selected were classified into an average of 10 different categories per literature review (SD: 4; $n=31$ ) versus an average of 33 categories (SD: $5 ; n=3$ ) with the updated process.

\section{Conclusions}

The new search method improves upon the previous method - it captures relevant articles indexed in health science and other sec- ondary databases beyond Medline. The new method has resulted in a greater number of relevant literature articles, from a broader range of disciplines, and in reduced amount of preparation time as compared to the results of the previous search method. This improvement may increase multi-disciplinary discussions and partnerships, but changes in online publishing pose challenges to continued access of the new range of articles.

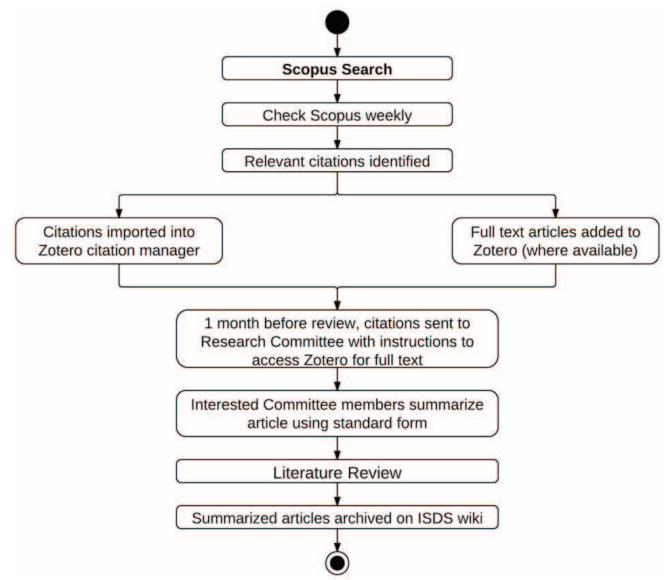

Figure. Overview of 2012 literature review process including, Scopus search [6]; Zotero [7], a freely available web application that streamlines content management; and summarized article archive on ISDS Wiki [8].

\section{Keywords}

Disease surveillance literature; ISDS Research Committee; Literature search

\section{Acknowledgments}

The ISDS RC would like to thank past contributors and guest authors to the literature review.

\section{References}

1. PubCrawler. http://www.webcitation.org/6AZiiSIDH

2. Google Scholar. http://www.webcitation.org/mainframe.php.

3. Pubmed. http://www.webcitation.org/6AZilS732.

4. Embase. http://www.webcitation.org/6AZj0rxhu.

5. Scopus. http://www.scopus.com/home.url.

6. ISDS Literature Review Search (Scopus). http://www.webcitation. org/6AZiWfC6C

7. Zotero. http://www.webcitation.org/6AZia0yEd.

8. ISDS Literature Summaries Archive. Wiki. http://www.webcitation. org/6AZkiT9xQ.

*Tera Reynolds

E-mail: treynolds@syndromic.org 